\title{
85-Year-Old Male with New Onset Melena
}

\author{
Benjamin D Shogan ${ }^{1,2^{*}}$ \\ ${ }^{1}$ Division of Colon and Rectal Surgery, Mayo Clinic, Rochester, Minnesota, USA \\ ${ }^{2}$ Section of Colon and Rectal Surgery, University of Chicago, Chicago, Illinois, USA
}

*Corresponding author: Benjamin D Shogan, Section of Colon and Rectal Surgery, University of Chicago, Chicago, IL, USA, E-mail: bshogan@surgery.bsd.uchicago.edu

\section{Case Report}

An 85-year-old otherwise healthy male originally presented to his local primary care physician with fatigue. He had no other complaints and specifically denied abdominal pain, constipation, diarrhea, or bloody stools. Routine lab work showed a hemoglobin of 7.5. To investigate his new onset anemia a colonoscopy was performed which found 3 large fungating masses in the sigmoid colon, transverse colon, and cecum. Because they were actively oozing, the masses were not biopsied, and for concern of active hemorrhage the patient was transferred to the local emergency department. The patient was admitted to the medical service and found to be hemodynamically stable, with no acute drop in hemoglobin. General surgery was consulted, and due to the reported active oozing, the patient was recommended to have a total abdominal colectomy. The patient and family were not comfortable with immediate surgical intervention and transferred care to a tertiary center.

The patient was evaluated by the colon and rectal surgery service upon arrival and a repeat history and physical was performed. The patient was confirmed to be hemodynamically stable. Repeat hemoglobin was stable at 8.2. A physical exam was unrevealing; his abdomen was soft and non-tender, and a digital rectal exam showed neither a mass nor gross blood. The patient reported a past surgical history of a right skin lesion excision in 2003, which upon further review of past records was found to be a stage $1 \mathrm{~b}$ malignant melanoma, without any adverse features. He denied any significant family history. Importantly, despite his age, his functional status was excellent; the patient was retired, lived with his wife on a farm, and performed all of his normal activities of daily living.
Based on the colonoscopic report the patient appeared to have a colonic malignancy of unknown etiology. Furthermore, finding 3 individual lesions questioned if this was a primary colonic malignancy, or an alternative diagnosis. He was therefore staged with a CT-chest/ abdomen/pelvis, which showed 3 colonic masses consistent with those found on colonoscopy, but no other abnormalities. Given his stability for over 24 hours without bleeding or transfusion, he was discharged, and an outpatient colonoscopy was scheduled in order to obtain a tissue diagnosis.

Two days later, the patient presented to the emergency room with bright red blood per rectum, tachycardia, and an acute drop in hemoglobin to 6.8. Despite resuscitation, the patient continued to have an ongoing lower gastrointestinal bleed, and therefore was brought urgently to the operating room. The patient underwent an exploratory laparotomy and subtotal colectomy with primary anastomosis. Final pathology revealed 4 ulcerated colonic masses, ranging from $2.1 \mathrm{~cm}$ to $8.0 \mathrm{~cm}$ consistent with undifferentiated high-grade malignant neoplasm, favoring malignant melanoma (Figure 1). Due to its undifferentiated cell type and lack of immunostaining a definitive melanoma could not be precisely confirmed but was assumed history and gross pathology. The patient had an unremarkable post-operative course and was discharged home.

\section{Discussion}

All melanomas, regardless of primary location, originate from melanocytes. Whereas cutaneous lesions are relatively common, melanoma of the gastrointestinal tract is a rare malignancy. Primary gastrointestinal mel-

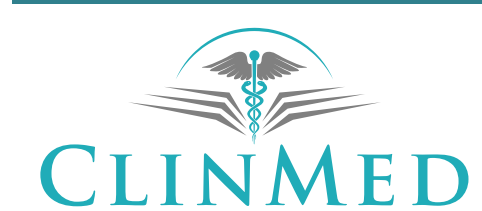

INTERNATIONAL LIBRARY
Citation: Shogan BD (2018) 85-Year-Old Male with New Onset Melena. Int J Surg Res Pract 5:078. doi. org/10.23937/2378-3397/1410078

Accepted: June 04, 2018; Published: June 06, 2018

Copyright: (C) 2018 Shogan BD. This is an open-access article distributed under the terms of the Creative Commons Attribution License, which permits unrestricted use, distribution, and reproduction in any medium, provided the original author and source are credited. 


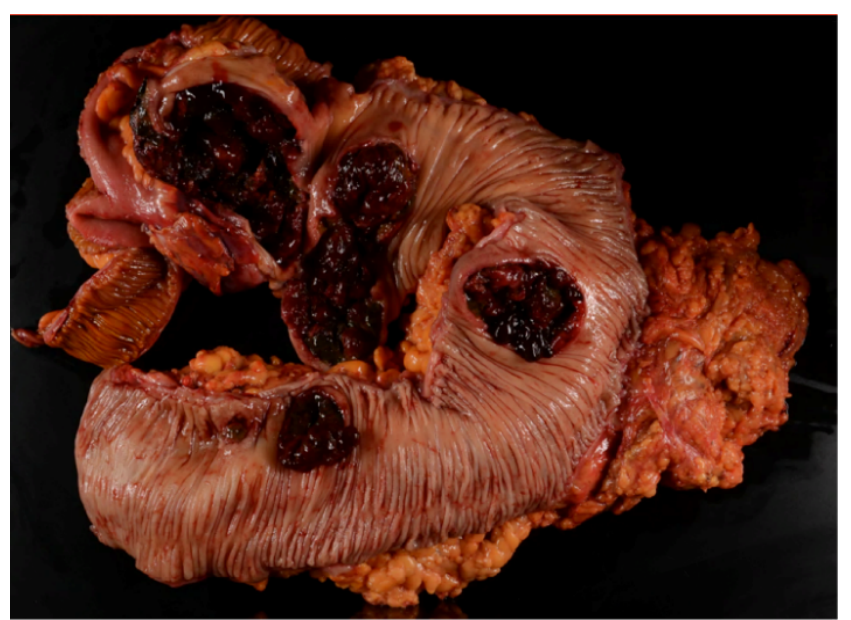

Figure 1: Gross pathology of resected colon showing 4 discrete lesions.

anoma can occur in the esophagus, small bowel, colon, rectum, and anus. Of the various locations, anorectal melanoma is the most common and has an annual incidence of 1-2 cases per 1 million people. When considering all types, anorectal melanoma represents $2 \%$ of all melanoma cases [1]. Population based studies report that gastrointestinal melanoma occurs equally in male and females, has a mean age of onset of 60-years-old, and likely has an equal distribution between Caucasian and African Americans [2].

The clinical presentation of gastrointestinal melanoma can greatly vary, often contributing to a significant delay in diagnosis. Most commonly they present as a bleeding anorectal mass resembling hemorrhoidal tissue, but ulcerations or large fungating masses have been described. When a potential lesion is found, a tissue biopsy is performed to confirm the diagnosis. Once the diagnosis is made, a staging protocol ensues to assess for regional and metastatic disease. Staging can be performed by either computerized tomography (CT) or MRI. On MRI, a high intensity T1 signal and mixed intensity T2 signal is consistent with a melanoma [3]. Positron emission tomography can also be used assess for distant disease, with a sensitivity and specificity ranging from $67-100 \%$, and may be superior to CT or MRI [4]. Melanoma is unfortunately an extremely aggressive disease and at the time of diagnosis, local regional and distant disease is found $60 \%$ and $30 \%$ of the time, respectively [1].

For those patients whom have no evidence of metastatic disease, surgical resection is the main stay of therapy. Colonic lesions can be treated with segmental resection, but controversy exists in the treatment of anal lesions. There have been multiple investigations comparing the need for local excision or abdominal perineal resection (APR) and there appears to be no significant benefit of aggressive excision [5]. In one such report from Memorial Sloan-Kettering in New York, the authors reported their 64-year experience in treating anorectal melanoma [6]. They report that in a cohort of 85 patients, those that underwent APR did have a trend toward increase 5-year survival compared to those with a local excision, but it did not reach statistical significance ( $27 \%$ APR vs. $5 \%$ local excision; $p=0.11$ ). Underscoring the aggressive nature of this disease, they reported a median survival of 19 -months and $17 \% 5$-year survival. Local excision provides the benefit of a significantly less morbid operation and quicker recovery. This is especially appealing given the aggressive nature of the neoplasm and that treatment, no matter how aggressive, may not significantly change patient outcomes [7].

More commonly than primary melanoma, melanoma can metastasize to the gastrointestinal tract from an alternative primary location. In an autopsy study of 216 patients with advanced melanoma, the authors found the incidence of gastrointestinal metastasis to be nearly 45\% [8]. In a similar series from Memorial Sloan Kettering in New York, they found that the incidence of gastrointestinal metastasis was small bowel (58\%), coIon (22\%), and rectum (5\%) [9]. Symptoms of metastasis can vary but most commonly include abdominal pain, bowel obstruction, perforation, and melana [10].

Prognosis of metastatic melanoma is especially poor with a median survival of only 7.5 months, and a 5 -year survival of only $6 \%$ [11]. Therefore, surgical intervention has largely been reserved for palliation. Contrary to what might be expected, though, several studies have shown a survival benefit in patients with metastatic melanoma to the gastrointestinal tract that undergo curative resection. In a review of 124 patients who underwent surgical resection of metastatic melanoma between 1971-1994, resection with curative intent had a median survival of 49 months whereas resection for palliation was only 5.4 months [12]. Multivariate analysis identified curative intent and gastrointestinal tract as the initial metastatic site as predictive of long-term survival. Some groups have therefore recommended surgical resection, not only for palliation, but for enhanced survival.

The patient described in this case report was found to have an undifferentiated high-grade malignant neoplasm. Although not conclusive for melanoma, the pathologist favored melanoma, and given the patients history of cutaneous melanoma, metastatic melanoma to the gastrointestinal tract remains in the differential. This case exemplifies the importance of a complete history and physical and reviews a rare colonic malignancy.

\section{References}

1. Singer M, Mutch MG (2006) Anal melanoma. Clin Colon Rectal Surg 19: 78-87.

2. Schuchter LM, Green R, Fraker D (2000) Primary and metastatic diseases in malignant melanoma of the gastrointestinal tract. Curr Opin Oncol 12: 181-185.

3. Matsuoka H, Nakamura A, Iwamoto K, Sugiyama M, Hachiya J, et al. (2005) Anorectal malignant melanoma: preoperative usefulness of magnetic resonance imaging. J Gastroenterol 40: 836-842. 
4. Prichard RS, Hill AD, Skehan SJ, O'Higgins NJ (2002) Positron emission tomography for staging and management of malignant melanoma. Br J Surg 89: 389-396.

5. Stefanou A, Nalamati SP (2011) Anorectal melanoma. Clin Colon Rectal Surg 24: 171-176.

6. Brady MS, Kavolius JP, Quan SH (1995) Anorectal melanoma. A 64-year experience at Memorial Sloan-Kettering Cancer Center. Dis Colon Rectum 38: 146-151.

7. Kohli S, Narang S, Singhal A, Kumar V, Kaur O, et al. (2014) Malignant melanoma of the rectum. J Clin Imaging Sci 4: 4.

8. Patel JK, Didolkar MS, Pickren JW, Moore RH (1978) Metastatic pattern of malignant melanoma. A study of 216 au- topsy cases. Am J Surg 135: 807-810.

9. Dasgupta TK, Brasfield RD (1964) Metastatic Melanoma of the Gastrointestinal Tract. Arch Surg 88: 969-973.

10. Liang KV, Sanderson SO, Nowakowski GS, Arora AS (2006) Metastatic malignant melanoma of the gastrointestinal tract. Mayo Clin Proc 81: 511-516.

11. Bhatia S, Tykodi SS, Thompson JA (2009) Treatment of metastatic melanoma: an overview. Oncology (Williston Park) 23: 488-496.

12. Ollila DW, Essner R, Wanek LA, Morton DL (1996) Surgical resection for melanoma metastatic to the gastrointestinal tract. Arch Surg 131: 975-979; 979-980. 